\title{
Algoritmo Matemático para Cuantificar Directamente el Ángulo de Convergencia en Preparaciones de Dientes Artificiales
}

\author{
Mathematical Algorithm to Directly Quantify the Convergence Angle in Tooth Preparations Dyes \\ ${ }^{*, * * *}$ Marco Flores; ${ }^{* *}$ Walter Lebrecht $\&$ *** Rodolfo Figueroa
}

FLORES, M.; LEBRECHT, W. \& FIGUEROA, R. Algoritmo matemático para cuantificar directamente el ángulo de convergencia en preparaciones de dientes artificiales. Int. J. Morphol., 29(4):1115-1122, 2011.

RESUMEN: El objetivo de este trabajo fue determinar un algoritmo matemático para cuantificar directamente el ángulo de convergencia (AC) en troqueles de preparaciones dentarias. El modelo experimental consistió en preparaciones coronarias simuladas sobre troqueles de yeso, en el cual el AC fue calculado por tres formulas trigonométricas. Las formulas fueron obtenidas de un modelo matemático en el cual la preparación coronaria representa una forma de pirámide truncada, la cual permite una proyección triangular en un plano. Fueron realizadas 60 mediciones in situ sobre las paredes de 15 troqueles. Se obtuvo una imagen de cada troquel usando una cámara digital (Schick ${ }^{\circ}$ CDR). El AC fue medido usando la herramientas del software (Control). Los datos fueron analizados estadísticamente y se aplicaron test de propagación de errores. Los ángulos calculados con las tres fórmulas matemáticas ([F1], [F2] y [F3]) mostraron un alto nivel de correlación con el grupo control excepto para dos muestras. Dentro de las limitaciones de este estudio podemos concluir que a través de este algoritmo matemático, es posible cuantificar directamente el AC de las preparaciones coronarias en troqueles. Actualmente la evaluación de los AC tanto en preparaciones realizadas por alumnos de pregrado de odontología como por dentistas, se hacen de manera subjetiva. Las tres fórmulas presentadas en el algoritmo tiene una correlación alta para cuantificar el AC en troqueles. La [F3], es la que mas correlación logra en todas las muestras $(0,89)$.

PAlabraS ClAVE: Prótesis; Corona dentaria; Preparación coronaria; Ángulo de convergencia; Modelo matemático.

\section{INTRODUCCIÓN}

En la odontología la confección de coronas periféricas, es la restauración más recomendada cuando existe una amplia destrucción coronaria, ya sea en dientes anteriores o posteriores. En los textos clásicos de prótesis dental, esta preparación debe tener características físicas de retención y resistencia al desalojo (Malone \& Koth 1989; Rosenstiel et al., 2001; Shillingburg et al., 1997). Uno de los principios fundamentales en la preparación dentaria es la forma de retención y resistencia (Malone \& Koth; Rosenstiel et al.; Shillingburg et al.). La capacidad y habilidad para obtener esta preparación es fundamental para el dentista. La hipótesis de que el ángulo de convergencia (AC) entre las superficies opuestas de la pared axial afecta la retención y la resistencia rotacional ha tenido considerable atención en la literatura (Trier et al., 1998; Potts et al., 1980; Parker et al., 1991; Wiskott et al., 1997). También esta convergencia es conocida en la literatura dental como "convergencia oclusal total" (Total Occlusal Convergence) (Goodacre et al., 2001) y está dada por el ángulo que se produce entre las paredes opuestas de la preparación dentaria para prótesis fija.

Estudios muestran una disparidad considerable de los registros de $\mathrm{AC}$ de las preparaciones y los recomendados por la literatura dental (Smith et al., 1999; Weed, 1980; Norlander et al., 1988; Ayad et al., 2005), considerando una ventaja alcanzar un adecuado ángulo de convergencia de la preparación con un mínimo de remoción dentaria (Ayad et al).

Una preparación dentaria con un ángulo de convergencia bajo o paredes lo más paralelas, tienen mayor retención y la preparación dentaria es más conservadora (Malone \& Koth; Rosenstiel et al.; Shillingburg et al.). Sin embargo, una preparación con paredes paralelas es dificultosa de lograr en la boca sin el riesgo de crear retenciones dentro de la preparación dentaria (Malone \& Koth; Rosenstiel et al.; Shillingburg et al.; Ohm \& Silness, 1978).

\footnotetext{
* Departamento de Odontología, Universidad de La Frontera, Chile.

** Departamento de Ciencias Físicas, Universidad de La Frontera, Chile.

${ }^{* * * *}$ Esta investigación fue financiada por el Proyecto de Investigación DIUFRO 2010. Iniciación a la Investigación No DI10-0008 y es parte de la Tesis Doctoral.
} 
Hay diferentes métodos indirectos que han sido usados para medir el AC de los dientes preparados y que incluyen herramientas de marcación con microscopio, microscopio goniométrico y retroproyectores (Smith et al.; Ayad et al.; Ohm \& Silness; Annerstedt et al., 1996). También, el AC puede ser medido usando una serie de imágenes fotocopiadas del mismo troquel con el cual se puede magnificar, para permitir una medición precisa (Noonan \& Goldfogel, 1991). Los software computacionales del tipo AutoCAD son un nuevo aporte tecnológico que puede medir el $\mathrm{AC}$ con gran precisión (Assunção et al., 2008a; Assunção et al., 2008b). Sin embargo, todos estos métodos de medición de ángulos son indirectos, y es necesaria la impresión de las preparaciones, y la confección de troqueles a los cuales se colocan en los instrumentos de medición o se digitalizan mediante fotografía. La forma para medir esta convergencia en clínica es sólo aproximada, usando una estimación visual o desarrollando una habilidad clínica para alcanzar el AC ideal (Nick et al., 2009; Parker et al., 2003). Okuyama et al. (2005) presentaron una evaluación cuantitativa del AC con un sofisticado sistema de medición láser 3D. En la literatura no se ha encontrado un método clínico simple y directo, que permita cuantificar el $\mathrm{AC}$ de manera directa $\mathrm{y}$ simple.

El propósito de este trabajo es desarrollar un método práctico y directo, para medir por cálculo trigonométrico, el $\mathrm{AC}$ en troqueles de preparaciones dentarias.

\section{MATERIAL Y MÉTODO}

Para el cálculo del AC, se ha considerado a la preparación dentaria como una figura con forma de pirámide truncada (Chan et al., 2007; Bowley et al., 2004), cuyas paredes laterales proyectan una figura geométrica, que a partir de la cual es posible obtener relaciones trigonométricas que nos llevan a determinar el AC por el triangulo proyectado. Esto constituye la primera parte de una investigación para cuantificar el AC in situ.

Específicamente hemos considerado como una proyección de un trapecio regular, tal que sus bases, siempre permanecen paralelas y sus lados modelan las paredes proximales (Fig. 1). En estas condiciones, se han establecido tres propuestas para calcular teóricamente el AC.

Para determinar el ángulo de convergencia de las paredes dentarias de un muñón de prótesis fija (corona) se ha desarrollado un algoritmo de cálculo asociando la longitud de las bases $\left(b_{1}\right.$ y $\left.b_{2}\right)$ y de los lados $\left(c_{1} y_{2} c_{2}\right)$ de esta preparación (Fig. 2).
Determinación del ángulo de convergencia mediante cálculo vectorial. Directamente se observa de la Fig. 2, la relación existente entre los vectores $\vec{b}_{2}$ y $\vec{c}_{2}$ y, por tanto:

$$
\vec{D}=\vec{b}_{2}+\vec{c}_{2}
$$

y como $\vec{b}_{2}=b_{2} \hat{i}$ entonces:

$$
\vec{c}_{2}=c_{2} \cos \left(90-\frac{\alpha}{2}\right) \hat{i}+c_{2} \operatorname{sen}\left(90-\frac{\alpha}{2}\right) \hat{j}
$$

La longitud del vector $\vec{D}$ se puede expresar como:

$$
\|\vec{D}\|^{2}=b_{2}{ }^{2}+c_{2}^{2}+2 b_{2} c_{2} \cos \left(90-\frac{\alpha}{2}\right)
$$

En forma análoga, ocupando los vectores $\vec{b}_{1}$ y $\vec{c}_{1}$ se obtiene que la longitud del vector $\vec{D}$ es:

$$
\|\vec{D}\|^{2}=b_{1}^{2}+c_{1}^{2}-2 b_{1} c_{1} \cos \left(90-\frac{\alpha}{2}\right)
$$

Se ha reportado la influencia que tiene la altura de la preparación o la pared lateral (paredes $c_{1}$ y $c_{2}$ ) sobre la resistencia y retención (Bowley et al., 2004; Al Ali et al., 2009). $\mathrm{Si}$ la altura ocluso gingival es preponderante, entonces podemos asumir que la pared de menor altura es la significativa, por tanto, se ha obtenido la $c_{1}$ y $c_{2}$ [F1] cuando, en ese caso igualando (3) y (4), se obtiene:

$$
\alpha=2 \operatorname{sen}^{-1}\left[\frac{\left(b_{1}-b_{2}\right)}{2 c}\right]
$$

Alternativamente considerando un promedio aritmético entre las paredes axiales $c_{1}$ y $c_{2}$, se ha obtenido la [F2], como:

$$
\alpha=2 \operatorname{sen}^{-1}\left(\frac{b_{1}-b_{2}}{c_{1}+c_{2}}\right)
$$

Por otro lado, considerando la situación asimétrica, se ha realizado un nuevo cálculo vectorial usando el mismo razonamiento descrito anteriormente, sobre un trapecio irregular (manteniendo el paralelismo entre $b_{1}$ y $b_{2}$ ), donde ahora $c_{1}$ y $c_{2}$ son distintos, y se ha obtenido la [F3] como:

$$
\alpha=\cos ^{-1}\left(\frac{c_{1}^{2}+c_{2}^{2}-\left(b_{1}-b_{2}\right)^{2}}{2 c_{1} c_{2}}\right)
$$

Aplicación de las fórmulas en Troqueles. Para la comprobación de las 3 fórmulas se han confeccionados 15 troqueles a los cuales se han preparado muñones tipo para prótesis fija, dejando las paredes axiales lo más paralela posible, tal como 


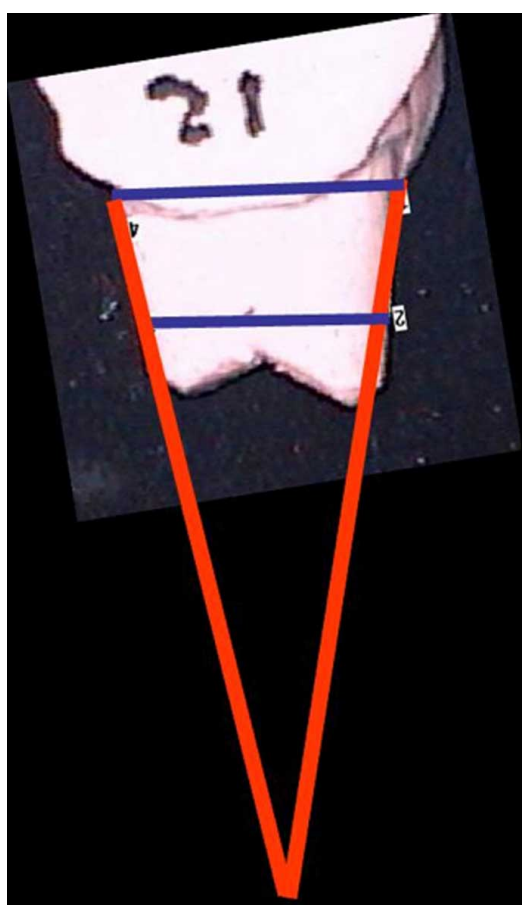

Fig. 1. Proyección de un trapecio regular. Vista lateral del troquel, se proyectan 2 líneas (rojas) de las paredes laterales (vestibular y palatino) formando un ángulo (Ángulo de convergencia). La línea azul superior corresponde a la distancia que une el inicio de la pared lateral tanto vestibular como palatino (base larga de un trapecio). La línea azul inferior corresponde a la unión del final de la pared lateral vestibular y palatino (base corta del trapecio).

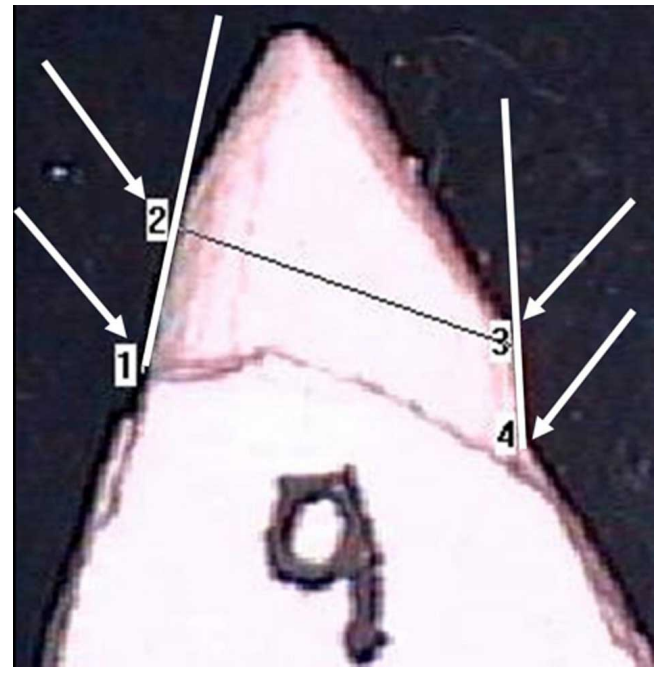

Fig.a 3. Zonas de medición del troquel. Los números indican donde de realizan las mediciones. 1 y 2 es la altura de la pared efectiva en la retención vestibular; 3 y 4 es la altura efectiva en la retención palatina; (ambas corresponden a c1 y c2). La distancia entre 1 y 4 es la base mayor, y entre 2 y 3 es la base menor de nuestro trapecio (b1 y b2).

lo indica el protocolo (Malone \& Koth; Rosenstiel et al.; Shillingburg et al.). En los troqueles (Fig. 3) se han marcado 2 puntos por la pared axial vestibular (n 1 y 2), y 2 puntos por pared palatina (n 3 y 4). Se midieron la altura de las paredes que dan la resistencia y retención de nuestra futura corona c1 (distancia entre 1 y 2) y c2 (distancia entre 3 y 4 ) - con un

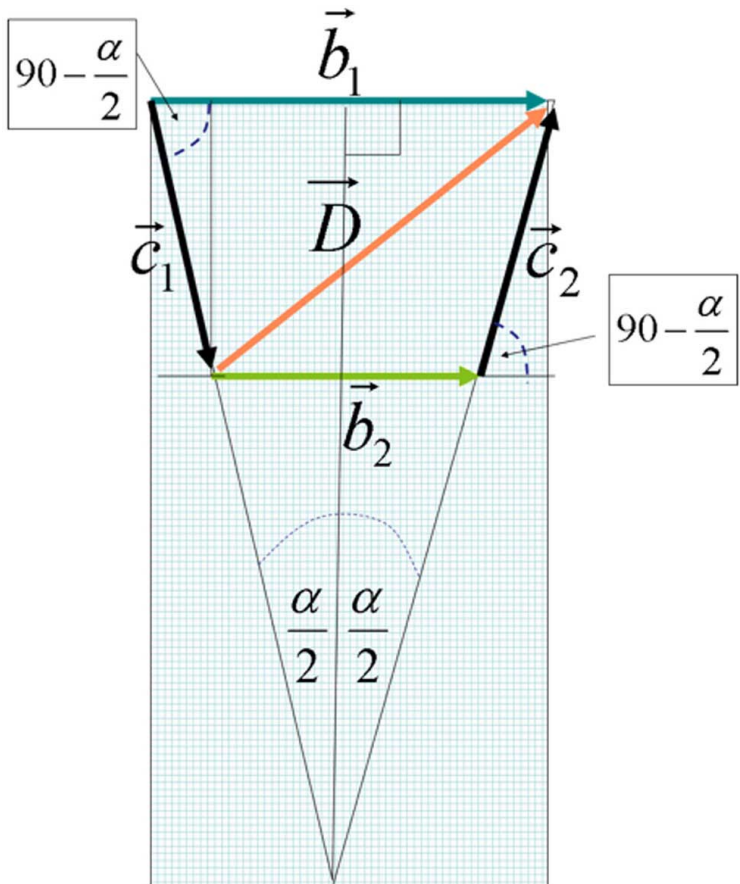

Fig. 2. Esquema vectorial del Algoritmo. Vista proximal de un troquel. c1 y c2 corresponde a la pared vestibular y palatina. b1 y b2 es la distancia de las bases (Base larga y base corta) en los extremos de las paredes c1 y c2. a = ángulo de convergencia.

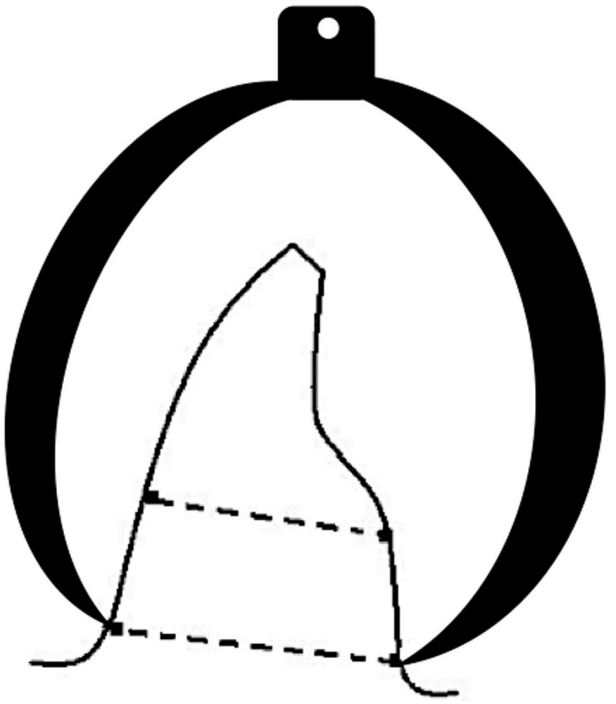

Fig. 4. Esquema de forma de medición. La base mayor y menor de miden con el calibrador milimétrico, manteniendo el paralelismo entre ambos.

pie de metro (Truper, Resolución 0,01mm). Posteriormente se midieron las longitudes b1 (distancia entre 1 y 4 ) y b2 (distancia entre 2 y 3), utilizando un calibrador de alta precisión (Schnelltaster, Resolución 0,01mm). La medición de las longitudes b1 y b2 se realiza de tal forma de mantener lo más paralelo el instrumento entre ambas longitudes (Fig. 4). 
Determinación del AC Control. Las paredes de cada troquel han sido fotografiado con una cámara intraoral (Schick ®) en donde se hicieron las mediciones. Se utilizó el software CDR

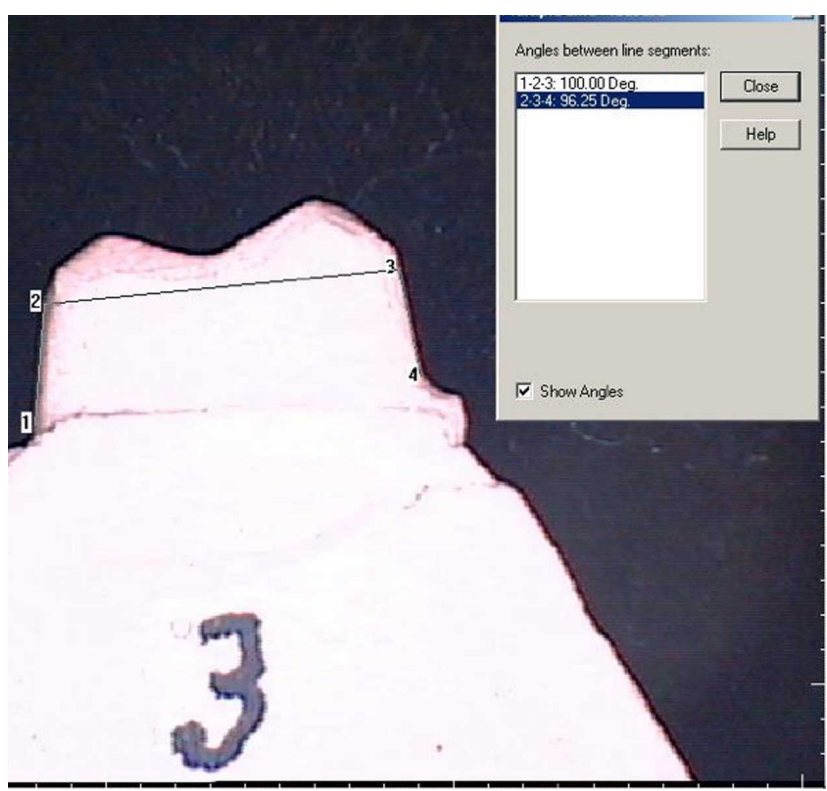

Fig. 5. Medición del AC control. El AC control se ha realizado con un software CDR para Windows de la cámara intraoral Schick®. El cálculo del AC control fue realizado mediante la construcción de trazos consecutivos que van uniendo la altura de las paredes c1 y c2. Con estos trazos se obtienen 2 ángulos internos que nos determina el software CDR (recuadro derecho mostrando los ángulos internos en grados). El AC Control de la muestra \#3 es la suma de los ángulos obtenidos menos $180^{\circ}\left(100,00+96,25-180=16,25^{\circ}\right)$. para Windows para calcular el AC control mediante la construcción de trazos consecutivos que van uniendo la altura de las paredes $\mathrm{c}_{1} \mathrm{y} \mathrm{c}_{2}$. Con estos trazos se obtienen 2 ángulos internos que nos determina el software CDR (recuadro derecho de Fig. 5), luego la suma de los ángulos obtenidos menos $180^{\circ}$ es el valor en grados de nuestro AC control en cada muestra.

Todos los datos han sido colocados en una Tabla Excel (Tabla I) y analizados estadísticamente para medir grado de acuerdo entre examinadores y el grado de correlación. Con el fin de asegurar la validez de los datos, se ha aplicado un test de propagación de errores para calcular el porcentaje relativo de error que podría incurrirse en el proceso de medición de las paredes del troquel.

\section{RESULTADOS}

En todos los ángulos calculados, se han encontrado valores menores a $18^{\circ}$, lo cual permite determinar que la preparación fue realizada dentro de los parámetros propuestos por la literatura (Tabla I, columna del ángulo control).

La mayoría de las muestras presentaron un resultado similar para las tres fórmulas, exceptuando las muestras 6 y 8. En estas muestras los valores entre $c_{1} y_{c_{2}}$ fueron muy distintos comparativamente (Fig. 6), por lo que [F3] se ajustó mejor al control debido a que individualiza ambos valores para obtener el AC.

Tabla I. Medición de la convergencia transversal.

\begin{tabular}{|c|c|c|c|c|c|c|c|c|}
\hline \multirow[t]{2}{*}{ Muestra } & \multicolumn{4}{|c|}{ Medición de Distancias en mm. } & \multicolumn{3}{|c|}{ A plicación de Fórmulas en grados } & \multirow{2}{*}{$\begin{array}{l}\text { Ángulo Control } \\
\text { en Grados }\end{array}$} \\
\hline & $\mathbf{b}_{1}$ & $\mathbf{b}_{2}<\mathbf{b}_{1}$ & $\mathbf{c}_{1}$ & $\mathbf{c}_{2}$ & [F1] & [F2] & [F3] & \\
\hline Troquel 1 & 8,08 & 7,46 & 2,01 & 2,07 & 17,744 & 17,481 & 17,400 & 17,3 \\
\hline Troquel 2 & 9,26 & 8,83 & 1,91 & 1,87 & 13,204 & 13,064 & 13,008 & 11,33 \\
\hline Troquel 3 & 8,57 & 8,02 & 2,25 & 2,41 & 14,041 & 13,556 & 12,975 & 16,25 \\
\hline Troquel 4 & 7,30 & 6,64 & 2,57 & 2,39 & 15,873 & 15,293 & 14,720 & 13,55 \\
\hline Troquel 5 & 7,37 & 6,62 & 2,75 & 2,61 & 16,521 & 16,087 & 15,808 & 17,83 \\
\hline Troquel 6 & 5,79 & 5,41 & 1,56 & 1,34 & 16,303 & 15,059 & 12,302 & 11,81 \\
\hline Troquel 7 & 5,91 & 5,51 & 1,76 & 1,71 & 13,433 & 13,239 & 13,136 & 12,5 \\
\hline Troquel 8 & 5,72 & 5,26 & 1,93 & 1,54 & 17,179 & 15,236 & 8,113 & 10,44 \\
\hline Troquel 9 & 5,69 & 5,50 & 1,53 & 1,58 & 7,120 & 7,005 & 6,759 & 8,37 \\
\hline Troquel 10 & 4,52 & 4,19 & 1,79 & 1,87 & 10,578 & 10,346 & 10,039 & 10,68 \\
\hline Troquel 11 & 6,14 & 5,87 & 2,07 & 2,01 & 7,702 & 7,589 & 7,400 & 9,86 \\
\hline Troquel 12 & 7,50 & 7,07 & 2,49 & 2,36 & 10,454 & 10,173 & 9,699 & 11,46 \\
\hline Troquel 13 & 8,50 & 8,31 & 2,43 & 2,58 & 4,481 & 4,347 & 2,669 & 5,66 \\
\hline Troquel 14 & 8,78 & 8,40 & 2,64 & 2,67 & 8,254 & 8,208 & 8,182 & 6,44 \\
\hline Troquel 15 & 4,99 & 4,53 & 1,79 & 1,72 & 15,369 & 15,061 & 14,888 & 17,27 \\
\hline
\end{tabular}




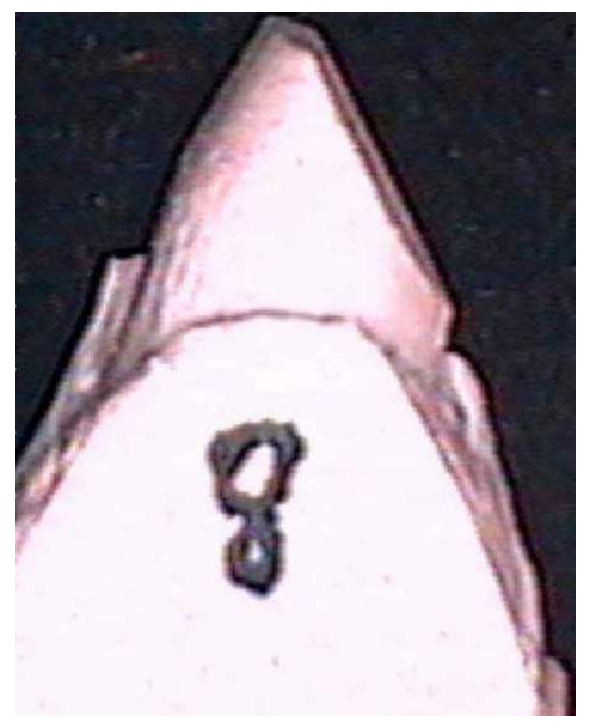

Fig. 6. Muestra de Troquel con gran diferencia entre $\mathrm{c}_{1} \mathrm{y} \mathrm{c}_{2}$. La pared palatina es bastante menor comparativamente a la pared vestibular. Esto podría explicar que el AC obtenido por la [F1] y [F2] estén alejados del AC control. Sin embargo, el AC obtenido por la [F3] es mucho mas cercano al control, debido a que identifica el valor independiente de $\mathrm{c}_{1} \mathrm{y} \mathrm{c}_{2}$.

Para evaluar el acuerdo entre examinadores se ha aplicado un análisis de confiabilidad, de las medidas obtenidas por las [F1], [F2] y [F3] con respecto a las medidas del grupo control (Coeficiente de correlación Intraclase). En las medidas de las tres fórmulas, el análisis de confiabilidad ha indicado un acuerdo de ellas con respecto al grupo control ( $p>0,05)$. Sin embargo, ha existido una mayor correlación en las medidas obtenidas por la [F3] $(0,89)$, luego la siguió la $[F 2](0,84)$ y finalmente la $[F 1](0,79)$ (Tabla II).

Los datos fueron sometidos a un test de propagación de errores, debido a que en el proceso de medición se debieron tomar 4 medidas para cada troquel $\left(\mathrm{b}_{1}, \mathrm{~b}_{2}, \mathrm{c}_{1}, \mathrm{c}_{2}\right)$.

Tabla II. Análisis de Confiabilidad.

\begin{tabular}{lcc}
\hline & Valor p>0,05 & $\begin{array}{c}\text { Coeficiente } \\
\text { Correlación } \\
\text { Intraclase }(\text { CCI) }\end{array}$ \\
\hline [F1] y el Control & 0,46824 & 0,79 \\
F2] y el Control & 0,90810 & 0,84 \\
[F3] y el Control & 0,06261 & 0,89 \\
\hline
\end{tabular}
errores).
Las fórmulas varían principalmente en la asignación del valor de la pared $c_{1} y_{c_{2}}$. Se ha asumido un rango de error para el $c_{1} y c_{2}$ de $0,1 \mathrm{~mm}$. por cada medición. Este rango fue calculado debido a que en cada medida realizada, el valor más crítico y difícil, según nuestro análisis, seria obtener la longitud del $\mathrm{c}_{1} \mathrm{y} \mathrm{c}_{2}$, luego el valor del rango fue influenciado por el promedio de la diferencias entre $c_{1}$ y $c_{2}(0,12 \mathrm{~mm})$. En cambio, el valor de la medida de las paredes representadas por $b_{1}, b_{2}$, es mas fácil obtener, y se le asignó la mitad de la dificultad de las otras paredes $(0,05 \mathrm{~mm})$. Con este análisis se obtiene el error absoluto y relativo para la medición de cada muestra. Esto se llevó a un gráfico por cada fórmula, mostrando que el porcentaje de error relativo, para este estudio, varió según la fórmula que ocupemos; siendo el rango de menor error relativo el de la fórmula 3 (Figs. 7, 8 y 9 y, Tablas III, IV y V de Propagación de

Error Relativo F 1

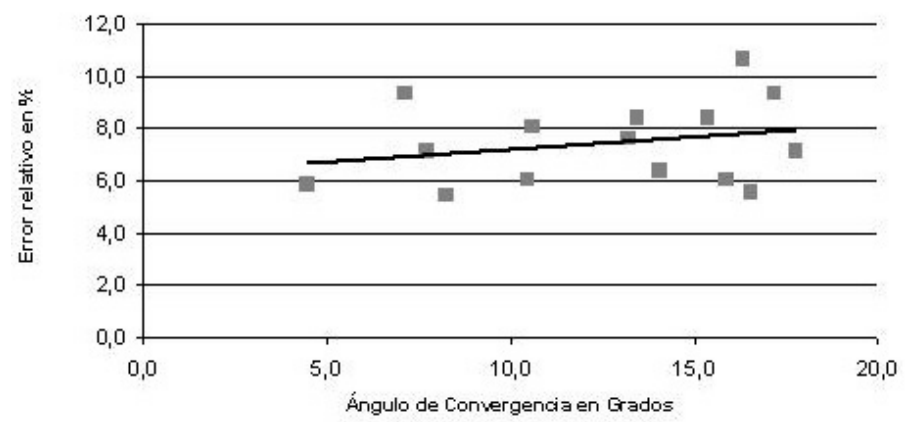

Fig. 7. Propagación de Errores para [F1].

Error Relativo F2

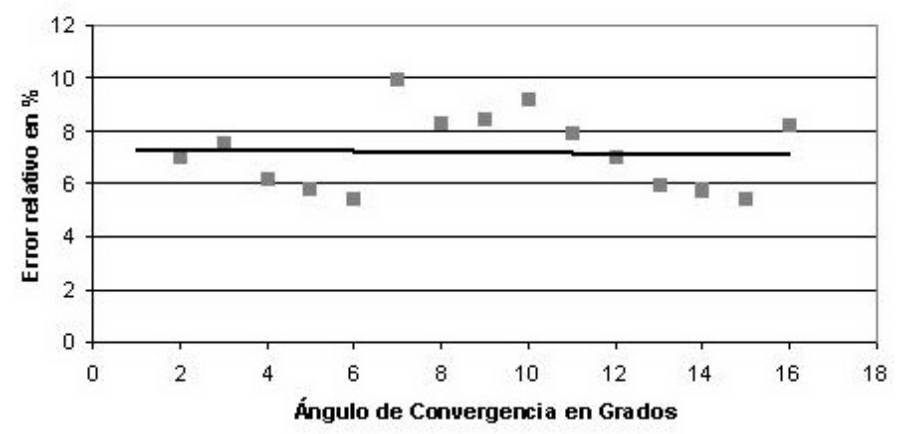

Fig. 8. Propagación de Errores para [F2].

Error Relativo F3

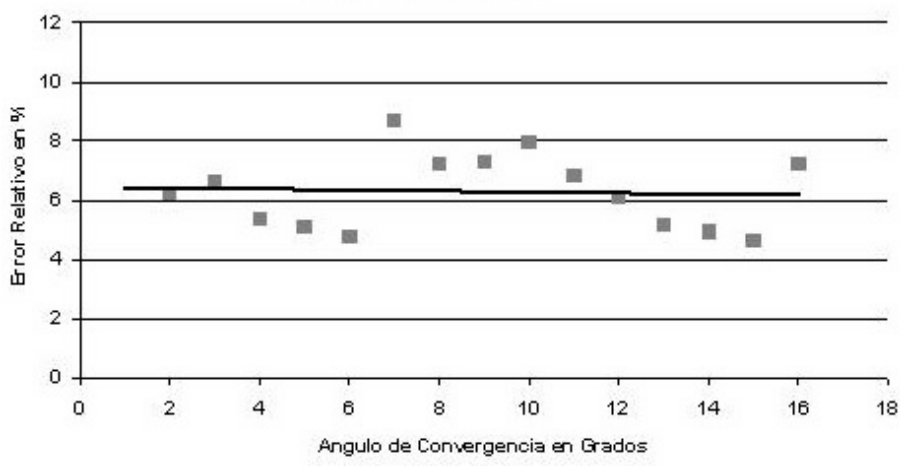

Fig. 9. Propagación de Errores para [F3]. 
Tabla III . Propagación de errores [F1].

\begin{tabular}{lccc}
\hline Muestra & Grados [F1] & $\begin{array}{c}\text { Error } \\
\text { absoluto }\end{array}$ & $\begin{array}{c}\text { Error } \\
\text { relativo \% }\end{array}$ \\
\hline Troquel 1 & 17,7 & 1,27 & 7,2 \\
Troquel 2 & 13,2 & 1,01 & 7,6 \\
Troquel 3 & 14,0 & 0,90 & 6,4 \\
Troquel 4 & 15,9 & 0,96 & 6,0 \\
Troquel 5 & 16,5 & 0,92 & 5,6 \\
Troquel 6 & 16,3 & 1,74 & 10,7 \\
Troquel 7 & 13,4 & 1,13 & 8,4 \\
Troquel 8 & 17,2 & 1,61 & 9,3 \\
Troquel 9 & 7,1 & 0,67 & 9,3 \\
Troquel 10 & 10,6 & 0,86 & 8,1 \\
Troquel 11 & 7,7 & 0,55 & 7,1 \\
Troquel 12 & 10,5 & 0,64 & 6,1 \\
Troquel 13 & 4,5 & 0,26 & 5,9 \\
Troquel 14 & 8,3 & 0,45 & 5,4 \\
Troquel 15 & 15,4 & 1,29 & 8,4 \\
\hline
\end{tabular}

Tabla IV . Propagación de errores [F2].

\begin{tabular}{lccc}
\hline Muestra & Grados [F2] & $\begin{array}{c}\text { Error } \\
\text { absoluto }\end{array}$ & $\begin{array}{c}\text { Error } \\
\text { relativo \% }\end{array}$ \\
\hline Troquel 1 & 17,5 & 1,23 & 7,0 \\
Troquel 2 & 13,1 & 0,99 & 7,6 \\
Troquel 3 & 13,6 & 0,84 & 6,2 \\
Troquel 4 & 15,3 & 0,89 & 5,8 \\
Troquel 5 & 16,1 & 0,87 & 5,4 \\
Troquel 6 & 15,1 & 1,50 & 10,0 \\
Troquel 7 & 13,2 & 1,10 & 8,3 \\
Troquel 8 & 15,2 & 1,29 & 8,5 \\
Troquel 9 & 7,0 & 0,64 & 9,2 \\
Troquel 10 & 10,3 & 0,82 & 7,9 \\
Troquel 11 & 7,6 & 0,53 & 7,0 \\
Troquel 12 & 10,2 & 0,60 & 5,9 \\
Troquel 13 & 4,3 & 0,25 & 5,7 \\
Troquel 14 & 8,2 & 0,44 & 5,4 \\
Troquel 15 & 15,1 & 1,24 & 8,2 \\
\hline
\end{tabular}

\section{DISCUSIÓN}

En esta investigación fueron utilizados troqueles de yeso con preparaciones de coronas periféricas, los cuales fueron medidos directamente.
Tabla V . Propagación de errores [F3].

\begin{tabular}{lccc}
\hline Muestra & Grados [F3] & $\begin{array}{c}\text { Error } \\
\text { absoluto }\end{array}$ & $\begin{array}{c}\text { Error } \\
\text { relativo \% }\end{array}$ \\
\hline Troquel 1 & 17,4 & 1,08 & 6,2 \\
Troquel 2 & 13,0 & 0,86 & 6,6 \\
Troquel 3 & 13,0 & 0,70 & 5,4 \\
Troquel 4 & 14,7 & 0,75 & 5,1 \\
Troquel 5 & 15,8 & 0,75 & 4,8 \\
Troquel 6 & 12,3 & 1,07 & 8,7 \\
Troquel 7 & 13,1 & 0,95 & 7,2 \\
Troquel 8 & 8,1 & 0,59 & 7,3 \\
Troquel 9 & 6,8 & 0,54 & 8,0 \\
Troquel 10 & 10,0 & 0,69 & 6,9 \\
Troquel 11 & 7,4 & 0,45 & 6,1 \\
Troquel 12 & 9,7 & 0,50 & 5,2 \\
Troquel 13 & 2,7 & 0,13 & 4,9 \\
Troquel 14 & 8,2 & 0,38 & 4,7 \\
Troquel 15 & 14,9 & 1,08 & 7,2 \\
\hline
\end{tabular}

La utilización de troqueles nos ha permitido situarnos de manera muy próxima a los futuros problemas que nos encontraremos cuando hagamos la medición in situ en boca de los pacientes.

El objetivo de esta primera investigación fue aplicar un algoritmo de medición para calcular el AC de una forma directa sobre el muñón del troquel. Los modelos representativos han sido usados por Nick et al., con el objetivo de determinar AC a través de una estimación visual. Su estudio no utilizó preparaciones in situ sino modelos representativos de dientes (typodont teeth), al igual que Smith et al., y Weed et al. Otros investigadores también han utilizado troqueles de yeso para estudiar la evaluación del AC y ha sido una alternativa práctica para las investigaciones que abordan el estudio comparativo del AC obtenido en preparaciones, realizadas por dentistas generales, especialistas o inclusive alumnos de odontología (Al Ali et al.; Dorriz et al., 2008; Pereira et al., 2008).

El estudio del AC ha tenido varios intereses en la literatura científica. Algunos han buscado asociación con el nivel de experiencia, ubicación del diente (anterior versus posterior) y unitaria versus plural (Al Ali et al.). Otros han buscado determinar el AC obtenidos por los estudiantes de odontología (Ayad et al.; Dorriz et al.; Pereira et al.) y compararlo entre si. También se ha investigado métodos para evaluar el $\mathrm{AC}$ a través de estimación visual (Nick et al.). Se han propuesto técnicas para alcanzar un AC adecuado (Parker et al., 2003). Y finalmente se ha realizado investigación asociando el AC a la cantidad de superficie dentaria (Chan et al.), a su 
resistencia a la carga (Cameron et al., 2006), y al complemento con surcos y cajas (Bowley \& Lai, 2007). Ninguno de estos estudios ha mostrado una forma de calcular el AC de las preparaciones de manera directa. Luego han determinado los ángulos en sus investigaciones, utilizando métodos indirectos como las fotografías de muñones, microscopio goniométrico, y escáner, pero en ninguno de ellos, el AC fue medido directamente del muñón.

Este trabajo muestra un algoritmo matemático para calcular el AC, basado en un método aplicando una fórmula trigonométrica a través de 4 medidas directas sobre el troquel. El método utilizó la medida de la altura de la pared axial (una a cada lado), y la medida del diámetro del muñón (uno basal y otro mas incisal) Fig. 3. Así, este método permite calcular el AC durante el proceso de la preparación, el clínico puede tomar los datos previos (alturas y diámetros) aplicar [F1], [F2] o [F3] y obtener el AC de la preparación.

Este trabajo es el primer reporte que ha indicado una correlación muy directa entre los datos que dan cualquiera de las 3 fórmulas, y más específicamente [F3], para el cálculo del AC directo de la preparación del troquel.

La [F3] ha mostrado una efectividad para la obtención del AC sin necesidad de elementos muy sofisticados como el Surflacer VMS-250R-H (Sistema de Medición de Forma 3D con rayo láser por Okuyama et al.).

Nuestro algoritmo se ha basado en el concepto que, el ángulo se obtiene por la proyección de las paredes axiales antagónicas (Fig. 1), y se condiciona a que los diámetros tanto basal e incisal deben estar paralelos entre si. Este último factor, es un elemento que explicaría las diferencias de los AC de las muestras \#6 y \#8 obtenidos por [F1] y [F2] y el control, versus el AC obtenido por [F3] que obtuvo una mayor correlación. Esto se ha debido a que en su fórmula ha considerado cierta asimetría de las paredes del muñón, y considerados como valores independientes de las paredes $\mathrm{c}_{1} \mathrm{y} \mathrm{c}_{2}$. Aun así, en la mayoría de las muestras, [F1], [F2] y [F3] han dado una buena correlación.

Dentro de las limitaciones de este estudio podemos concluir que a través de este algoritmo matemático, es posible cuantificar el AC sobre troqueles directamente. Actualmente la evaluación de los AC tanto en preparaciones realizadas por alumnos de pregrado de odontología como por dentistas, se hacen de manera subjetiva.

Las tres fórmulas presentadas en el algoritmo tiene una correlación alta para cuantificar el AC en troqueles. La [F3], es la que mas correlación logra en todas las muestras.

Son necesarios estudios adicionales que validen este método in situ, sobre preparaciones dentarias en pacientes.

\section{AGRADECIMIENTOS}

Los autores agradecen al Sr. Antonio Sanhueza del Departamento de Matemáticas de la Universidad de La Frontera, por su tiempo dedicado en la revisión del análisis estadístico.

FLORES, M.; LEBRECHT, W. \& FIGUEROA, R. Mathematical algorithm to directly quantify the convergence angle in tooth preparations dyes. Int. J. Morphol., 29(4):1115-1122, 2011.

SUMMARY: The aim of this study was to determine a mathematical algorithm to directly quantify the convergence angle (AC) dyes tooth preparations. The experimental model system consisted of crown preparations dyes simulated on plaster, which the CA was quantify by three trigonometric formulas. Formulas were obtained from mathematical models representing the crown preparation as a truncated pyramid shape, allowing a triangular projection on the plane. 60 direct measurements were made in situ on the walls of 15 dyes. An image was obtained from each dye using a digital camera (Schick ${ }^{\circledR}$ CDR). The CA were measured using software tools (Control). The data were statistically analyzed and test data were applied to propagation of errors. The angles calculated with the three math formulas ([F1], [F2] and [F3]) showed a high level of correlation with the control except for two samples. Within the limitations of this study we can conclude that through this mathematical algorithm, it is possible to directly quantify the AC preparation coronary dyes. Currently the evaluation of AC in both preparations made by undergraduate students of dentistry as well as dentists, are made subjectively. The three formulas presented in the algorithm have a high correlation to quantify the AC dyes. The [F3], correlation is the most accomplished in all samples.

KEY WORDS: Prosthodontics; Tooth crown; Tooth preparations; Convergence angle; Mathematical modeling.

\section{REFERENCIAS BIBLIOGRÁFICAS}

Al Ali, K.; Al Wazzan, K.; Al Amri, M.; Al-Shahrani, A.; Al-Shahrani, M. \& Al-Qahtani H. Assessment of convergence angle of full veneer preparations carried out by practitioners with different levels of experience. Saudi Dent. J., 21(1):37-44, 2009. 
Annerstedt, A.; Engstrom, U.; Hansson, A.; Jansson, T.; Karlsson, S.; Liljhagen, H.; Lindquist, E.; Rydhammar, E.; TyremanBandhede, M.; Svensson, P. \& Wandel, U. Axial wall convergence of full veneer crown preparations. Documented for dental students and general practitioners. Acta Odontol. Scand., 54(2):109-12, 1996.

Assunção, W. G.; Gomes, E. A.; Tabata, L. F. \& Gennari-Filho, H. A comparison of profilometer and AutoCAD software techniques in evaluation of implant angulation in vitro. Int. J. Oral Maxillofac. Implants, 23(4):618-22, 2008a.

Assunção, W. G.; Tabata, L. F.; Cardoso, A.; Rocha, E. P. \& Gomes, E. A. Prosthetic transfer impression accuracy evaluation for osseointegrated implants. Implant Dent., 17(3):248-56, 2008b.

Ayad, M. F.; Maghrabi, A. A. \& Rosenstiel, S. F. Assessment of convergence angles of tooth preparations for complete crowns among dental students. J. Dent., 33(8):633-8, 2005.

Bowley, J. F.; \& Lai, W. T. Surface area improvement with grooves and boxes in mandibular molar crown preparations. J. Prosthet. Dent., 98(6):436-44, 2007.

Bowley, J. F; Sun, A. F. \& Barouch, K. K. Effect of margin location on crown preparation resistance form. J. Prosthet. Dent., 92(6):546-50, 2004.

Cameron, S. M.; Morris, W. J.; Keesee, S. M.; Barsky, T. B. \& Parker, M. H. The effect of preparation taper on the retention of cemented cast crowns under lateral fatigue loading. J. Prosthet. Dent., 95(6):456-61, 2006.

Chan, D. C.; Chan, B. H. \& Chung, A. K. Mathematical modeling of molar tooth preparations for complete crowns. J. Dent., 35(11):875-7, 2007.

Dorriz, H.; Nokar, S.; Baghai Naini, R. \& Madadi, A. The Convergence Angle of Full-coverage Crown Preparations Made by Dental Students. J. Dent. Tehran Univ. Med. Sci., 5(1):37-41, 2008.

Goodacre, C. J.; Campagni, W. V.; \& Aquilino, S. A. Tooth preparations for complete crowns: an art form based on scientific principles. J. Prosthet. Dent., 85(4):363-76, 2001.

Malone, W. F. \& Koth, D. L. Tylman's Theory and Practice of Fixed Prosthodontics. 8th ed. St Louis, CV Mosby, 1989. pp.2-45.

Nick, D. R.; Clark, M.; Miler, J.; Ordelheide, C.; Goodacre C. \& Kim J. The ability of dental students and faculty to estimate the total occlusal convergence of prepared teeth. J. Prosthet. Dent., 101(1):7-12, 2009.

Noonan, J. E. Jr. \& Goldfogel, M. H. Convergence of the axial walls of full veneer crown preparations in a dental school environment. J. Prosthet. Dent., 66(5):706-8, 1991.
Norlander, J.; Weir, D.; Stoffer, W. \& Ochi, S. The taper of clinical preparations for fixed prosthodontics. J. Prosthet. Dent., 60(2):148-51, 1988.

Ohm, E. \& Silness, J. The convergence angle in teeth prepared for artificial crowns. J. Oral Rehabil., 5(4):371-5, 1978.

Okuyama, Y.; Kasahara, S. \& Kimura, K. Quantitative evaluation of axial wall taper in prepared artificial teeth. J. Oral Sci., 47(3):129$33,2005$.

Parker, M. H.; Ivanhoe, J. R.; Blalock, J. S.; Frazier, K. B. \& Plummer, K. D. A technique to determine a desired preparation axial inclination. J. Prosthet. Dent., 90(4):401-5, 2003.

Parker, M. H.; Malone, K. H. 3rd; Trier, A. C. \& Striano, T. S. Evaluation of resistance form for prepared teeth. J. Prosthet. Dent., 66(6):730-3, 1991.

Pereira, P.; Lima, S. I.; Mochel, J.R. \& Travassos, J.R. Avaliação do ângulo de convergência de preparos para coroas totais. Rev. Fac. Odont. Univ. Passo Fundo, 13(1):43-7, 2008.

Potts, R. G.; Shillingburg, H. T. Jr. \& Duncanson, M. G. Jr. Retention and resistance of preparations for cast restorations. J. Prosthet. Dent., 43(3):303-8, 1980.

Rosenstiel, S. F.; Land, M. F. \& Fujimoto, J. Contemporary fixed prosthodontics. $3^{\text {th }}$ ed. St. Louis, CV Mosby, 2001. pp.166-201.

Shillingburg, H. T.; Hobo, S. \& Whitsett, L. D. Fundamentals of Fixed Prosthodontics. $3^{\text {th }}$ ed. Chicago, Quintessence Publishing, 1997. pp.119-20.

Smith, C. T.; Gary, J. J.; Conkin, J. E. \& Franks, H. L. Effective taper criterion for the full veneer crown preparation in preclinical prosthodontics. J. Prosthodont., 8(3):196-200, 1999.

Trier, A. C.; Parker, M. H.; Cameron, S. M. \& Brousseau, J. S. Evaluation of resistance form of dislodged crowns and retainers. J. Prosthet. Dent., 80(4):405-9, 1998.

Weed, R. M. Determining adequate crown convergence. Tex. Dent. J., 98(5):14-6, 1980.

Wiskott, H. W.; Nicholls, J. \& Belser, U. The effect of tooth preparation height and diameter on the resistance of complete crowns to fatigue loading. Int. J. Prosthodont., 10(3):207-15, 1997.

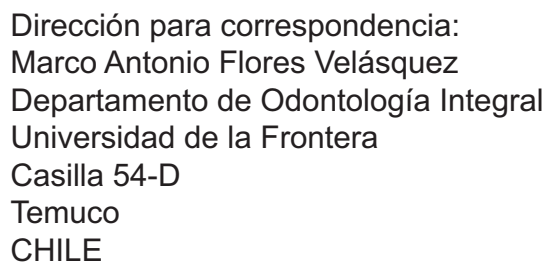

Email: drmarcoflores@gmail.com

Recibido : 11-07-2011

Aceptado: 08-08-2011 